\title{
Necessary detection efficiencies for secure quantum key distribution and bound randomness
}

\author{
Antonio Acín, ${ }^{1,2}$ Daniel Cavalcanti, ${ }^{1}$ Elsa Passaro, ${ }^{1}$ Stefano Pironio, ${ }^{3}$ and Paul Skrzypczyk ${ }^{1,4}$ \\ ${ }^{1}$ ICFO-Institut de Ciencies Fotoniques, Mediterranean Technology Park, 08860 Castelldefels (Barcelona), Spain \\ ${ }^{2}$ ICREA-Institució Catalana de Recerca i Estudis Avançats, Lluis Companys 23, 08010 Barcelona, Spain \\ ${ }^{3}$ Laboratoire d'Information Quantique, Université Libre de Bruxelles (ULB), Bruxelles, Belgium \\ ${ }^{4}$ H. H. Wills Physics Laboratory, University of Bristol,Tyndall Avenue, Bristol, BS8 1TL, United Kingdom.
}

(Dated: March 12, 2018)

\begin{abstract}
In recent years, several hacking attacks have broken the security of quantum cryptography implementations by exploiting the presence of losses and the ability of the eavesdropper to tune detection efficiencies. We present a simple attack of this form that applies to any protocol in which the key is constructed from the results of untrusted measurements performed on particles coming from an insecure source or channel. Because of its generality, the attack applies to a large class of protocols, from standard prepare-and-measure to device-independent schemes. Our attack gives bounds on the critical detection efficiencies necessary for secure quantum distribution, which show that the implementation of most partly device independent solutions is, from the point of view of detection efficiency, almost as demanding as fully device-independent ones. We also show how our attack implies the existence of a form of bound randomness, namely non-local correlations in which a non-signalling eavesdropper can find out a posteriori the result of any implemented measurement.
\end{abstract}

Over the past few decades the problem of bridging the gap between realistic implementation of Quantum Key Distribution (QKD) protocols and their theoretical security proofs has attracted a lot of attention. The security of standard QKD protocols [1, 2] relies on a very detailed modeling of the preparing and measuring devices. However, unavoidable imperfections of the devices or unnoticed failures lead in practice to deviations from the model used to prove security - deviations that can be taken advantage of by a potential eavesdropper. Indeed, standard QKD protocols, being dependent on the accuracy with which the devices are described, can typically suffer attacks, for instance on the detectors [3].

To overcome these problems a new paradigm was proposed, adopting the device-independent (DI) framework [4]: In this scenario no assumptions are made either on the source of the shared system or on the internal working of the devices, which are treated like "black boxes". In this context the only object one relies on is the statistics of inputs and outputs, and the security of a device-independent quantum key distribution (DIQKD) protocol is guaranteed by the nonlocal character of these statistics [5]. The DI scenario allows for the most general and powerful quantum certification protocols as it depends on very few assumptions. Nevertheless, their implementations are demanding because they require very high detection efficiencies to close the detection loophole (e.g. with photonic implementations [5, 6]).

In order to make the experimental implementations less demanding other scenarios between standard and fully DI QKD have been introduced. In these intermediate scenarios the parties involved add some extra assumptions to the fully-DI scheme. The focus is still on the input/output statistics but with an intermediate level of trust between the fullyDI framework and the device-dependent one. For instance, semi-device-independent (SDI) protocols have been proposed where one makes an assumption on the dimension of the involved quantum systems but, apart from this assumption, the devices are still uncharacterized [7]. From an implementation point of view, the advantage of SDI protocols is that they do not require entanglement and can be implemented in a prepare-and-measure configuration. Another class of intermediate scenario, known as one-sided device-independent (1SDI) [8-10], is based upon quantum steering [11] which consists of a bipartite scenario in which one of the parties trusts his measuring devices but the other does not.

All these different QKD solutions are based on different assumptions and, thus, offer different levels of security. Although different QKD protocols use different strategies, most of them share the property that the key is constructed from the results of measurements performed by one of the end-users on quantum particles that have propagated through an insecure channel. This is the case, for instance, of the famous Bennett-Brassard-84 [1] and Ekert [2] protocols, and standard DIQKD protocols, such as those introduced in [4, 12]. Notice however that not every QKD protocol is of this form, a paradigmatic example being measurementdevice-independent QKD [13, 14].

In this work, we consider the above scenario and therefore focus on an end-user in a cryptographic protocol who performs measurements on some quantum systems received through an insecure channel. We introduce an attack by an eavesdropper who is able to control the detection efficiency of the measurements - a natural assumption in the adversary model of cryptographic protocols based on untrusted measurements, such as 1SDI, SDI, and DI protocols. The attack also applies to standard prepare-and-measure protocols if one cannot guarantee that the eavesdropper is unable to tune the detection efficiencies. In fact recent hacking attacks on standard QKD protocols have exploited the ability to manipulate detection efficiencies [3]. Our attack defines detection efficiencies necessary for secure quantum key distribution using the previous protocols. We then discuss how our attack can also be applied to schemes for randomness generation. From a practical point of view, our results imply that the implementation of partly DI protocols are, in terms of detection efficiency, almost as demanding as fully DI ones. Moreover, our attack has also implications from a fundamental point of view: as also observed independently in [15, 16], it implies the existence of a very weak form of intrinsic randomness in which an 
eavesdropper limited only by the no-signalling principle [17] cannot a priori fix the outputs of the measurements in a Bell test, but she can later find out the result of any implemented measurement. In analogy with results in thermodynamics and entanglement theory [18] we name this effect bound randomness.

\section{THE ATTACK}

The considered scenario consists of a party, say Bob, who measures quantum systems received through an insecure channel. The received systems may have been prepared by another honest party, say Alice, or by an untrusted source. In particular, they may be entangled with other quantum systems. Bob performs on them one of $M_{B}$ possible measurements with $D$ possible outcomes. We label the measurement choice and result by $y=1, \ldots, M_{B}$ and $b=1, \ldots, D$ respectively. In the absence of loss, let Bob's device give the outcome $b$ with probability $Q(b \mid y, \rho)$, where $\rho$ is the state of the system received by Bob and which may be correlated with classical or quantum variables of other parties in the protocol. For simplicity in the notation, we omit $\rho$ in what follows, as our results are independent of it.

In a realistic implementation with losses and inefficient detectors, each measurement of Bob will have a detection efficiency $\eta_{y}$, and one more outcome is observed, corresponding to the no-click events which we denote by $b=\emptyset$. That different measurements may have different efficiencies naturally arises in certain situations, e.g. in [19]. In such a situation, Bob's device then produces outcomes with probabilities $P(b \mid y)=\eta_{y} Q(b \mid y)$ for $b=1, \ldots, D$, and $P(\emptyset \mid y)=1-\eta_{y}$.

We exhibit here below a simple attack which allows Eve to learn the output of any subset $G \in\left\{1, \ldots, M_{B}\right\}$ of Bob's measurements. This attack does not modify any of Bob's outcome probabilities, i.e., it reproduces the full lossy behavior of Bob's device. In particular, we stress that it does not rely on Bob performing any kind of post-selection. The attack requires that Eve is able to tune arbitrarily the detection efficiency of Bob's detectors depending on the implemented measurement and works as long as Bob's observed detector efficiencies satisfy $\sum_{y \in G} \eta_{y} \leq 1-\eta^{\prime}$, where $\eta^{\prime}=\max _{y \notin G} \eta_{y}$ is the maximum detection efficiency over the set of measurements complementary to $G$, i.e., those that Eve is not interested in guessing. (If this complementary set of measurements is empty, i.e. when Eve wants to guess the output of all of Bob's measurement, we define $\eta^{\prime}=0$ ).

In the simple case where all detectors have the same efficiency $\eta_{y}=\eta$, the attack works whenever $\eta \leq 1 /(|G|+1)$ if $|G|<M_{B}$ or when $\eta \leq 1 / M_{B}$ if $|G|=M_{B}$. In particular, when Eve is interested in guessing a single one of Bob's measurements, say $\bar{y}$, then $|G|=1$ and the attack works as long as $\eta \leq 1 / 2$. Furthermore, if the detectors are not all equally efficient, Eve can use the inefficiency of the measurements $y \neq \bar{y}$ that she is not interested in to raise the critical efficiency of the measurement $\bar{y}$ that she wants to guess above $\eta_{\bar{y}}=1 / 2$, as long as $\eta_{\bar{y}} \leq 1-\max _{y \neq \bar{y}} \eta_{y}$.

Let us now explain how the attack works. Eve randomly selects with probability $\eta_{y}$ one of the measurement $y \in G$ whose outcomes she wants to guess and with probability $1-\sum_{y \in G} \eta_{y}$ she does not select any particular measurement. Depending on her choice, she then applies one of the two following strategies.

(i) If she picked measurements $\bar{y} \in G$, she performs this measurement on the incoming state. She obtains outcome $b$ with probability $Q(b \mid \bar{y})$, she reads the outcome, and forwards the corresponding reduced state to Bob. On Bob's side, she forces Bob's detector to click if he performs measurement $y=\bar{y}$, in which case he obtains the same outcome $b$. If otherwise $y \neq \bar{y}$, she instructs Bob's device not to click, i.e., to output $b=\emptyset$.

(ii) If she didn't select any particular measurement, she directly forwards the state to Bob without intervention. However, she instructs Bob's device not to click $(b=\emptyset)$ if $y \in G$. If on the other hand $y \notin G$, she allows his detector to click with probability $\tau_{y}$. Bob then obtains a proper result $b$ with probability $\tau_{y} Q(b \mid y)$ and a no-click result with probability $1-\tau_{y}$.

Obviously, Eve can always correctly guess Bob's output when $y \in G$ since when Bob's measuring device clicks, it always coincides with Eve's previous measurement result, and she always knows when his detector does not click (gives outcome $b=\emptyset)$. Moreover, defining the $\tau_{y}$ such that $\eta_{y}=(1-$ $\left.\sum_{y \in G} \eta_{y}\right) \tau_{y}$ for $y \notin G$, it is straightforward that the strategy yields the overall outcome probabilities $P(b \mid y)=\eta_{y} Q(b \mid y)$ if $b \neq \emptyset$ and $P(\emptyset \mid y)=1-\eta_{y}$, which correspond to lossy devices characterized by detection efficiencies $\eta_{y}$. The only requirement for the $\tau_{y}$ s to be well-defined is that $\sum_{y \in G} \eta_{y} \leq 1-\eta^{\prime}$, where $\eta^{\prime}=\max _{y \notin G} \eta_{y}$.

\section{A. Application to QKD protocols}

The above attack applies to any cryptographic protocol in which the key is constructed from the results of measurements performed by one of the end-users on quantum particles received through an insecure channel. It thus applies to any Bell based DI protocol, but also to SDI approaches where the dimension is fixed, protocols based on steering, or prepare-andmeasure protocols, unless the eavesdropper cannot tune Bob's detection efficiencies. In fact, in many of these protocols, the key is constructed from a single measurement, which means that in the best case scenario (that of equal detection efficiencies) they become insecure at $\eta=1 / 2$. It is important to notice that the obtained critical detection efficiencies apply to any scenario, independently of the number of measurements $M_{B}$, outputs $D$, or the role of other parties in the protocol.

By using many measurements for the key generation, one increases the number of measurements that Eve needs to guess and the critical detection efficiency for our attack decreases. However, this solution is demanding from Alice's and Bob's point of view as many more symbols are sacrificed after basis reconciliation, and also more statistics needs to be collected to have a proper estimation of the protocol parameters. In fact the advantage of using more measurements is limited when considering two distant parties connected by a lossy channel. Take for instance a rather idealised situation in which all 
losses come from the channel, denoted by $\eta_{C}$ and are equal to $\eta_{C}=10^{\frac{-\alpha L}{10}}$ where $L$ is the distance in $\mathrm{km}$. Then, the improvement in distance with the number of bases is only logarithmic. For instance, assuming a typical value for the losses of $\alpha$ of the order of $0.2 \mathrm{~dB} / \mathrm{km}$, one has that in order to compensate for the channel losses at $100 \mathrm{~km}$ Alice and Bob need to employ 100 bases.

A possible solution to overcome channel losses is to use heralded schemes [20, 21] or quantum repeaters based on entanglement swapping [22]. Using such schemes, which are technologically more demanding, the only relevant losses for security are those on the honest parties' labs. Alice and Bob can then decide which cryptographic solution to adopt, from standard to fully device-independent, depending on the observed detection inefficiencies and the plausibility of the assumptions needed for security.

Our attack also applies to randomness generation schemes based on correlations between measurements on two different devices. In these schemes, randomness is certified by the observed quantumness of the correlations, certified for instance by means of steering [23, 24] or Bell inequalities [25, 26]. As the particles come from a untrusted source, one cannot exclude that the attack has been implemented on each of the particles sent to the untrusted parties in the protocol (one in the case of steering and two for Bell-based schemes).

In the case of Bell-based protocols, for instance, it is possible to guess the result of one measurement on each device when their detection efficiency is $1 / 2$. Note that in the context of randomness expansion, it is usually the case that one of the possible combinations of measurements is implemented most of the time, as this requires much less initial randomness to run the Bell test [26]. For all these protocols, randomness expansion is lost when the critical detection efficiency is $1 / 2$.

\section{B. Improved attacks}

The previous attack applies to many cryptographic scenarios because it is independent of the number of measurements, outputs and actions by other parties. Improvements however may be expected for concrete protocols. For instance, we show in what follows how for two untrusted measuring devices, Eve can improve the attack by exploiting the detection efficiency of the second party too. Note though that the attack needs more operations from Eve's side on the untrusted devices than just varying the detection efficiency of the implemented measurements. This improved attack is inspired by the local models exploiting detection inefficiencies introduced in [27].

We thus consider a second party in the protocol, Alice, who performs $M_{A}$ measurements of $D$ outputs. Her measurement choice and result are labeled by $x$ and $a$. Again, in the presence of loss, the output probability distribution has one more result because of the no-click events and is of the form $P(a b \mid x y)=\eta^{2} Q(a b \mid x y), P(\emptyset b \mid x y)=\eta(1-\eta) Q(b \mid y)$, $P(a \emptyset \mid x y)=\eta(1-\eta) Q(a \mid x), P(\emptyset \emptyset \mid x y)=(1-\eta)^{2}$, where the detection efficiencies have for simplicity all been taken to be equal to $\eta$.
In the improved attack, Eve's goal is again to guess $G$ measurements on Bob's side. With probability $q$ Eve uses the previous attack and does nothing on Alice's side. With probability $1-q$ the attack works in the reverse direction: Eve fixes the output of one of Alice's measurements (even though she is still guessing Bob's result). That is, she picks one of Alice's measurements, say $\bar{x}$, with probability $1 / M_{A}$, and decides an output for this measurement following the quantum probability $Q(a \mid \bar{x})$. If Alice happens to implement measurement $\bar{x}$ she will obtain this outcome, otherwise she observes a no-click. On Bob's side, Eve computes the reduced state corresponding to Alice's result and, for each measurement by Bob, selects one possible outcome following the probability $Q(b \mid y, a x)$ predicted by this state. This defines Bob's result, whose detector always clicks. The intuition behind the attack is that for those cases in which Eve fixes Alice's result, she can allow any measurement on Bob to give a result, as Alice effectively implements one single measurement and a hidden-variable model is enough to describe the observed correlations.

So far the model never gives two no-click events, which does not correspond to the expected behavior of actual lossy devices. To correct this, with probability $r$, Eve runs the above protocol and with probability $1-r$, she instructs both detectors not to click. We finally get

$$
\begin{aligned}
& P(a b \mid x y)=r\left(\frac{q}{|G|^{\prime}}+\frac{1-q}{M_{A}}\right) Q(a b \mid x y) \\
& P(a \emptyset \mid x y)=r q\left(1-\frac{1}{|G|^{\prime}}\right) Q(a \mid x) \\
& P(\emptyset b \mid x y)=r(1-q)\left(1-\frac{1}{M_{A}}\right) Q(b \mid y) \\
& P(\emptyset \emptyset \mid x y)=1-r=(1-\eta)^{2},
\end{aligned}
$$

where $|G|^{\prime}=|G|+1$ when $|G|<M_{B}$ and $|G|^{\prime}=|G|$ when $|G|=M_{B}$, as in the previous attack. Tuning the parameters so that the above probabilities correspond to those of lossy devices with equal efficiencies $\eta$, one finds

$$
\eta=\frac{|G|^{\prime}+M_{A}-2}{|G|^{\prime} M_{A}-1} .
$$

It is easy to see that this attack improves over the previous one, as the corresponding critical detection efficiency is always larger than $1 /|G|^{\prime}$. For example, in the simplest case where Alice performs 3 measurements, Bob performs two, and Eve guesses a single outcome, $\left(M_{A}, M_{B},|G|\right)=(3,2,1), \eta=$ $3 / 5$, increasing the critical efficiency by a further $10 \%$. In the opposite limit, when $M_{A} \rightarrow \infty, \eta \rightarrow 1 /|G|^{\prime}$, showing that the advantage of attacking Alice's measurements decreases with the number of measurements she performs.

\section{BOUND RANDOMNESS}

Our results are not only limited to practical aspects of cryptographic protocol implementations, but also have implications from a more fundamental point of view. Indeed, they imply the existence (see also [15, 16]) of non-local correlations 
with a very weak form of randomness in which an eavesdropper (i) cannot obviously fix the results of all measurements in advance but (ii) can later predict with certainty the outcome of any measurement. As mentioned, we dub this effect bound randomness. Our last result is to show the existence of bound randomness in the case of eavesdroppers limited only by the no-signalling principle [17].

The construction of bound randomness relies on a couple of simple observations. First, in a randomness scenario consisting of two untrusted devices with uniform detection efficiency $\eta=1 / 2$, our (primary) attack can be applied to both parties, so that the eavesdropper learns the result of one measurement each for Alice and Bob, $\bar{x}$ and $\bar{y}$. Let $e=\left(e_{a}, e_{b}\right)$ be Eve's prediction for Alice and Bob's outcomes for measurements $\bar{x}$ and $\bar{y}$. This variable can take $(D+1)^{2}$ possible values corresponding to the ideal $D$-valued measurement outcomes plus the no-detection event. Eve obtains outcome $e$ with a certain probability $P_{\bar{x} \bar{y}}(e)$ and given $e$, her attack defines a joint probability $P_{\bar{x} \bar{y}}(a b \mid x y, e)$ for Alice and Bob. Since the attack does not change the expected probabilities $P(a b \mid x y)$ from Alice and Bob's perspective, we have that

$$
\sum_{e} P_{\bar{x} \bar{y}}(a b e \mid x y)=P(a b \mid x y)
$$

where we have defined the tripartite conditional probability distribution $P_{\bar{x} \bar{y}}(a b e \mid x y)=P_{\bar{x} \bar{y}}(e) P_{\bar{x} \bar{y}}(a b \mid x y, e)$. Now, the $M_{A} M_{B}$ different attacks defined by each combination of measurement settings $z=(\bar{x}, \bar{y})$ can be combined into a single tripartite conditional probability distribution

$$
P(a b e \mid x y z) \equiv P_{z}(a b e \mid x y)
$$

by adding an input $z$ on Eve's, where $z$ defines the combination of settings Eve wants to predict. It is easily verified that this tripartite distribution is no-signalling, see also [28], and thus represents a valid attack by a no-signalling eavesdropper. By choosing her input $z$, Eve can steer the ensemble of non-signalling correlations prepared between Alice and Bob. Thus, she can choose a posteriori the attack that allows her to predict the result of any given pair $z$ of implemented measurements. The effect is similar to what happens in the quantum case when predicting the result of non-commuting variables on half of a maximally entangled state.

Note now that there exist correlations that are non-local hence whose outcomes cannot all be fixed in advance - even when the detection efficiency is smaller than $1 / 2$ - hence whose outcomes can all be perfectly guessed by Eve a posteriori using the above construction. Examples of such correlations were given in [29], where it was shown that the critical detection efficiency required to close the detection loophole decreases exponentially with the dimension of the measured quantum state in a scenario in which the number of measurements by Alice and Bob is exponentially large. More generally, any non-local correlations obtained for detection efficiencies $\eta \leq 1 / 2$ constitute examples of bound randomness. Finally, it can be explicitly checked that both the all-versus nothing example of [30] and the Peres-Mermin magic square [31] exhibit bound randomness [32].

\section{CONCLUSIONS}

We have provided a simple and general detection attack that allows an eavesdropper to guess some of (or all) the measurement results in a cryptographic protocol. It applies basically to any protocol with untrusted detectors in which she is able to tune the detection efficiency of untrusted devices. Obviously our attack cannot be applied to protocols in which the key is not constructed from measurement results, such as in measurement-device-independent schemes [13, 14]. These protocols, almost by definition, are only sensitive to attacks on the devices that prepare the quantum states. The generality of our attack also implies that the implementation of partly DI solutions is, from the point of view of detection efficiency, almost as demanding as DI ones, which, in turn, offer stronger security.

Interestingly, the critical detection efficiency corresponding to our attack only depends on the number of measurements that Eve wants to learn, but is independent of the total number of measurements $M_{B}$, number of outputs $D$, or dimension of the quantum systems used.

We have also presented an improved attack that applies to protocols with two untrusted detectors. In this attack, the eavesdropper exploits the detection inefficiencies of one of the parties to improve her attack on the other party. More generally, it would be interesting to derive a formalism to study the robustness of concrete protocols to detection attacks, as these are the most advanced at the moment. This will allow us to understand for which protocols the detection bounds for security derived here are tight. An analysis of the tightness of our attack in steering scenarios will be presented in [24].

Finally, our results imply also the existence of a bound randomness, an intriguing and weak form of certified randomness. In a scenario in which an eavesdropper is limited only by the no-signalling principle, there exist non-local correlations for which she can find out a posteriori the results of any implemented measurements. A final open question is to understand if this form of randomness exists in the quantum case, that is, when the eavesdropper is limited by the quantum formalism.

\section{ACKNOWLEDGMENTS}

Acknowledgments. We thank M. Pawłowski for useful discussions. This work was supported by the Spanish project FOQUS, the Generalitat de Catalunya (SGR 875), the ERC CoG QITBOX and the John Templeton Foundation, the EU project QALGO, by the F.R.S.-FNRS under the project DIQIP, the Brussels-Capital Region through a BB2B grant and the Interuniversity Attraction Poles program of the Belgian Science Policy Office under the grant IAP P7-35 photonics @ be. DC is supported by the Beatriu de Pinós fellowship (BP-DGR 2013) and PS partially by the Marie Curie COFUND action through the ICFOnest program and the ERC AdG NLST. SP is a Research Associate of the Fonds de la Recherche Scientifique F.R.S.-FNRS (Belgium). 
[1] C. H. Bennett and G. Brassard, in Proceedings of the IEEE International Conference on Computers, Systems and Signal Processing (IEEE Press, New York, 1984) pp. 175179.

[2] A. K. Ekert, Phys. Rev. Lett. 67, 661 (1991).

[3] L. Lydersen et al., Nature Phot. 4, 686 (2010); I. Gerhardt et al., Nature Comm. 2, 349 (2011).

[4] A. Acín, N. Brunner, N. Gisin, S. Massar, S. Pironio, V. Scarani, Phys. Rev. Lett. 98, 230501 (2007). S. Pironio, A. Acin, N. Brunner, N. Gisin, S. Massar, V. Scarani, New J. Phys. 11, 045021 (2009).

[5] N. Brunner, D. Cavalcanti, S. Pironio, V. Scarani and S. Wehner, Rev. Mod. Phys. 86, 419 (2014).

[6] M. Giustina et al., Nature 497, 227 (2013); B.G.Christensen et al., Phys. Rev. Lett. 111, 130406 (2013).

[7] M. Pawłowski and N. Brunner, Phys. Rev. A 84, 010302 (2011).

[8] M. Tomamichel and R. Renner, Phys. Rev. Lett. 106, 110506 (2011).

[9] M. Tomamichel, C. C. W. Lim, N. Gisin, and R. Renner, Nat. Commun. 3, 634 (2012).

[10] C. Branciard, E. G. Cavalcanti, S. P. Walborn, V. Scarani, and H. Wiseman, Phys. Rev. A 85, 010301 (2012).

[11] H. M. Wiseman, S. J. Jones, and A. C. Doherty, Phys. Rev. Lett. 98, 140402 (2007).

[12] A. Acín, S. Massar and S. Pironio, New J. Phys. 8, 126 (2006).

[13] H.-K. Lo, M. Curty and B. Qi, Phys. Rev. Lett. 108, 130503 (2012).

[14] S. L. Braunstein and S. Pirandola, Phys. Rev. Lett. 108, 130502 (2012).

[15] E. Woodhead, PhD Thesis, Universit Libre de Bruxelles, December 2014, available at http://theses.ulb.ac.be/ETD-db/collection/available/ULBetd-12092014-07491

[16] E. Woodhead, S. Pironio, J. Silman, in preparation.

[17] J. Barrett, L. Hardy and A. Kent, Phys. Rev. Lett. 95, 010503 (2005).

[18] M. Horodecki, P. Horodecki, and R. Horodecki, Phys. Rev. Lett. 80, 5239 (1998).

[19] D. Cavalcanti, N. Brunner, P. Skrzypczyk, A. Salles and V. Scarani, Phys. Rev. A 84, 022105 (2011).

[20] N. Gisin, S. Pironio and N. Sangouard, Phys. Rev. Lett. 105, 070501 (2010).

[21] A. Máttar, J. Brask and A. Acín, Phys. Rev. A 88, 062319 (2013).

[22] J. Hofmann et al., Science 3376090 (2012).

[23] Y. Z. Law, L. P. Thinh, J.-D. Bancal and V. Scarani, J. Phys. A: Math. Theor. 47, 424028 (2014).

[24] E. Passaro, P. Skrzypczyk, D. Cavalcanti, A. Acín, New J. Phys. 17, 113010 (2015).

[25] R. Colbeck, PhD Thesis University of Cambridge, arXiv:0911.3814 (2006).

[26] S. Pironio et al., Nature 464, 1021 (2010).

[27] S. Massar and S. Pironio, Phys. Rev. A 68, 062109 (2003).

[28] E. Hänggi, R. Renner and S. Wolf, Theor. Comp. Sci. 486, 27 (2013).

[29] S. Massar, Phys. Rev. A 65, 032121 (2002).

[30] A. Cabello, Phys. Rev. Lett. 87, 010403 (2001).

[31] P. K. Aravind, Am. J. Phys. 72, 1303 (2004).

[32] E. Hänggi, private communication. 